\title{
Interface Modification for Tuning the Contact Resistance of Metal/ Organic Semiconductor Junctions
}

\author{
Gilles Horowitz*
}

ITODYS, Université Denis-Diderot, 15 rue Jean-Antoine de Baïf, 75205 Paris cedex 13, France

\begin{abstract}
As the performance of organic field-effect transistors improves, the limitation due to charge carrier injection at source and drain electrodes becomes crucial. This review describes the various solutions that have been developed to work around this issue. The most widespread method consists of interposing between the electrodes and the organic semiconductor film a self-assembled monolayer made of an appropriate reactive molecule. In that case, the reduction of the contact resistance may come either from an improved morphology of the semiconductor film, or to a better energy level alignment at the interface with the electrode. The respective inference of both aspects is discussed. Alternative ways to reduce the contact resistance by an appropriate surface treatment of the electrodes prior to the deposition of the semiconductor are also presented.
\end{abstract}

Keywords: Organic electronics, organic field-effect transistors, self-assembled monolayers.

\section{INTRODUCTION}

Organic electronics opens new opportunities to the development of a new generation of semiconductor devices featured by low cost, flexibility, low weight and low power consumption. Several applications have been foreseen, spanning from displays and lighting (organic light emitting diodes, OLEDs) to solar energy conversion (organic photovoltaic cells, OPVs) [1]. Within the general domain of electronics, the organic field-effect transistor (OFET) holds a pivotal position, because transistors at large are the central element of all electronic circuits. Devices that have received attention as potential applications for OFETs include smart cards, radio frequency identification (RFID) tags, electronic paper and backplane circuitry for active matrix displays. All of them benefit from the ability of OFETs to be integrated into flexible, large area devices, and also from the low-cost and little energy demanding fabrication processes. Prototypes of new products have been unveiled, and some of them are currently close to their introduction into the market.

Since the beginning of OFETs, 25 years ago, continuous efforts have been devoted to the synthesis of air stable, high mobility semiconductors based on small molecules and polymers. A remarkable breakthrough in the recent years has been the synthesis of air-stable, soluble, high-mobility n-type organic semiconductors based on perylene derivatives, which paves the way to the achievement of an organic complimentary circuitry $[2,3]$.

However, as performance improves, interfaces tend to play a role as crucial as materials in the overall performance of the devices. There are two kinds of interfaces in a

*Address correspondence to this author at the ITODYS, Université DenisDiderot, 15 rue Jean-Antoine de Baif, 75205 Paris cedex 13, France; Tel: +33 15727 5430; Fax: +331 5727 7263;

E-mail: horowitz@univ-paris-diderot.fr transistor; that between the semiconductor and the gate dielectric, where the conducting channel forms, and that between the source and drain electrodes and the semiconductor film, where charge carriers are injected and retrieved. The present review focuses on the latter. We will in particular discuss the still controversial issue of what is the predominant mechanism that controls charge carrier injection at the source electrode, morphology or energy level alignment.

\section{BASIC CONCEPTS}

\section{Operation of the Field-Effect Transistor}

Most organic transistors adopt the so-called insulatedgate geometry, which can be visualized as a capacitor where one of the plates is constituted by the organic semiconductor film. The other plate is the gate electrode that controls the current flowing in the device. The semiconductor film is equipped with two additional electrodes, the source and the drain, whose function is to inject and remove charge carriers into the semiconductor. Because an organic semiconductor essentially behaves as an electrical insulator, practically no current flows in the semiconductor film when a voltage $V_{D}$ is applied between source and drain. However, applying an appropriate voltage $V_{G}$ to the gate results in the charging of the capacitor, thus leading to charge injection at the semiconductor-insulator interface, and a substantial increase of the source-drain current. In most cases, the organic transistor is a unipolar device (it works with one kind of charge carriers); an $n$-channel transistor works with electrons and a $p$ channel transistor with holes. The device is in its on state when $V_{G}$ is positive for an $n$-channel device (negative for a $p$-channel device).

The various configurations that the three elements of the organic transistor can adopt are depicted in Fig. (1).

One can distinguish two regimes in the current-voltage characteristic of an organic transistor: The linear regime, 


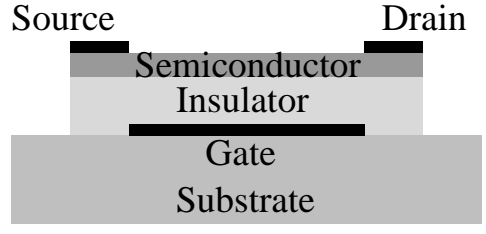

Bottom gate, top contacts

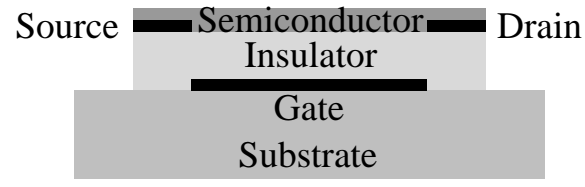

Bottom gate, bottom contacts

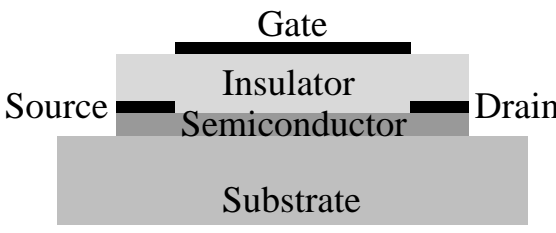

Top gate, top contacts

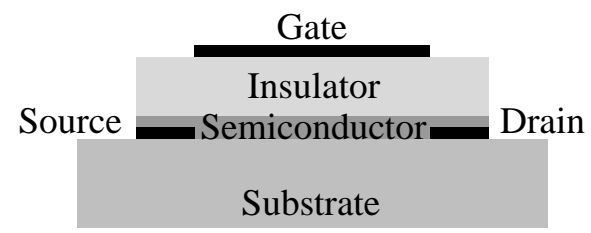

Top gate, bottom contacts

Fig. (1). Cross-sectional view of the various configurations an organic transistor can adopt.

which is observed at low source-drain voltages, and the saturation regime, when the source-drain voltage exceeds the gate voltage. The modification of the electrical potential (and in turn the charge density) profile alongside the channel under application of a voltage to the drain explains this behavior. The linear regime dominates as long as the source-drain voltage is lower than the gate voltage. When $V_{D}$ increases up to $V_{G}$, the potential at a given point (close to the drain electrode) of the channel falls to zero, the channel is pinched off and the drain current saturates. The drain current in the linear and saturation regimes is described by Eqs. (1) and (2), where $W$ and $L$ are the width and length of the channel, $\mu$ the mobility of charge carriers, $C_{i}$ the capacitance (per unit area) of the gate dielectric and $V_{T}$ the threshold voltage.

$$
\begin{aligned}
& \mathrm{I}_{\mathrm{D}}=\frac{\mathrm{W}}{\mathrm{L}} \mu \mathrm{C}_{\mathrm{i}}\left(\mathrm{V}_{\mathrm{G}}-\mathrm{V}_{\mathrm{T}}-\frac{\mathrm{V}_{\mathrm{D}}}{2}\right) \mathrm{V}_{\mathrm{D}}, \\
& \mathrm{I}_{\text {Dsat }}=\frac{\mathrm{W}}{2 \mathrm{~L}} \mu \mathrm{C}_{\mathrm{i}}\left(\mathrm{V}_{\mathrm{G}}-\mathrm{V}_{\mathrm{T}}\right)^{2} .
\end{aligned}
$$

Fig. (2) shows representative characteristics of an nchannel transistor. The main parameter that controls the performance of the device is the charge carrier mobility $\mu$, which can be extracted from the transfer characteristic. Other important parameters are the on/off ratio, and the subthreshold slope that measures the switching capability of the device.

\section{The Metal-Semiconductor Interface}

The performance of an organic transistor not only relies on the way charge carriers are transported along the conducting channel, but also on how they are injected into the semiconductor. This is largely because charges that intervene in the process of charge transport practically exclusively come from the electrodes. This means, for an n-channel device, injection of electrons into the LUMO level, and for a pchannel device, injection of holes into the HOMO level. Ideally, contacts should be ohmic; that is, capable of providing any kind of charge carrier with negligible voltage drop. In practice, and in contrast to what found in silicon, contacts in organic transistors result of a direct metal-semiconductor junction, without any doping of the semiconductor. Under such circumstances, holes and electrons experience an injection barrier that correspond to the difference between the HOMO and LUMO levels and the Fermi level of the metal, as shown in Fig. (3).

In reality, the picture given in Fig. (3) may be substantially modified by the presence of an interface dipole that induces a shift of the vacuum level at both side of the interface $[5,6]$. The origins of the dipole are diverse. Its effect is particularly important when the Fermi level approaches the edges of the HOMO-LUMO gap. For this reason, the injection barriers cannot get below a minimum value that can be estimated to around $0.2 \mathrm{eV}$. The mechanism of this "Fermi a)

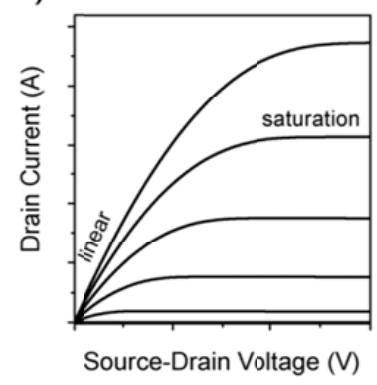

b)

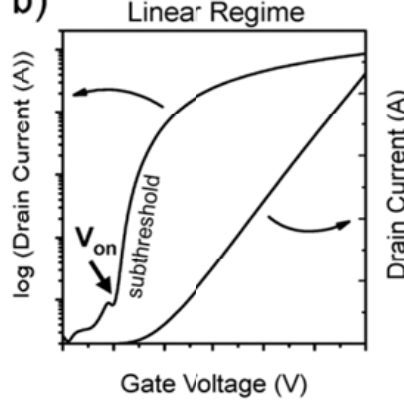

C) Saturation Regime

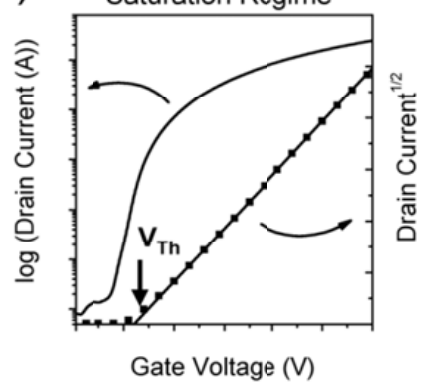

Fig. (2). Representative current-voltage characteristics of an n-channel organic field-effect transistor. (a) output characteristics with indication of the linear and saturation regimes; (b) transfer characteristic in the linear regime $\left(V_{D}<V_{G}\right)$; $V_{\text {on }}$ is the onset voltage, where the drain current increases abruptly; (c) transfer characteristic in the saturation regime $\left(V_{D}>V_{G}-V_{T}\right) ; V_{T}$ is the threshold voltage, which can be extracted from a linear fitting of the square root of the drain current (adapted from Ref. [4]). 


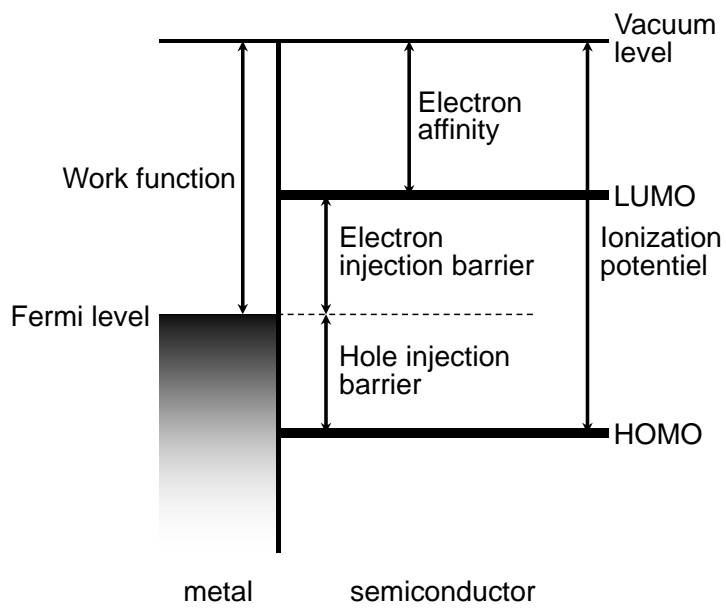

Fig. (3). Energy level alignment at a metal-semiconductor interface. The electron (hole) injection barrier is given by the difference between the electron affinity and the Fermi level of the metal (Fermi level and ionization potential).

level pinning" is depicted in Fig. (4) [7]. Here, $W_{m}$ is the electrode work function and $\Delta$ the interface dipole.

As will be detailed in the following, an elegant way to control the injection barrier is to purposely introduce an appropriate interface dipole. This can be done for instance with the help of a suitable self-assembled monolayer.

\section{Morphological Effects}

Another source of contact resistance has been identified from the early stage of organic electronics. It comes from morphology rather than energy level alignment. Direct evidence for the role of morphology is given by the importance of the device architecture on its final performance. Topcontact transistors (see Fig. 1) have better performance in terms of mobility and threshold voltage over bottom contact devices [8]. This can be understood by thinking of depositing a layered crystal structure such that of pentacene over a substrate that presents different surface properties. Fig. (5) illustrates the dramatic effect the transition between the electrode and the dielectric can induce in a bottom contact configuration. The diagram shows two types of disorder introduced in a short molecule layer as its growth is confronted with a discontinuity in the surface energy of the substrate. On the low-

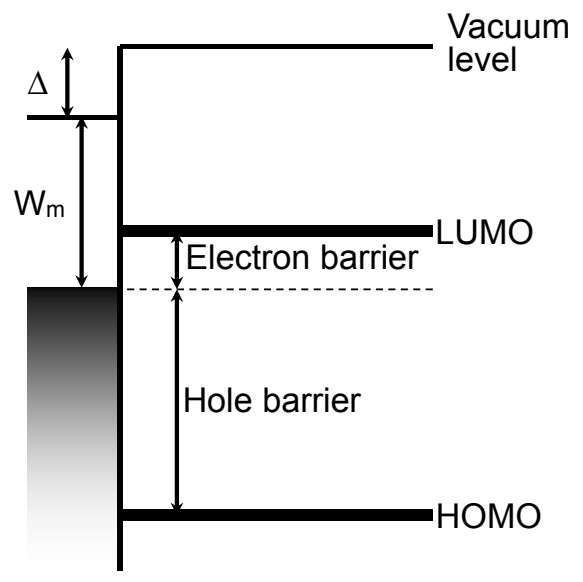

energy-surface dielectric, the molecules tend to align straight on the surface, while they lay down on the metal high-energy surface [9]. The step existing at the metal-dielectric transition reinforces this tendency to disorder. There are two ways to work around this issue: (1) realizing a planar structure where the electrode and the insulator would be on the same level. Such a solution poses huge technological problems that have not been all solved to date; [10] (2) modifying the electrode surface to decrease its surface energy. This has been done with the help of self-assembled monolayers [1113].

The top-contact configuration does not suffer this problem. Here, the organic semiconductor is deposited on a homogeneous planar surface where the molecules can adopt a well-ordered configuration over the whole area. However, this architecture most often limits to large channel distances because the use of photolithography is problematic, because of the sensitivity of the organic semiconductor to process chemicals.

\section{SELF-ASSEMBLED MONOLAYERS}

\section{Production}

After the first description of gold-alkylthiolate selfassembled monolayers (SAM) by Allara and Nuzzo in 1983, many groups initiated works on the preparation and characterization of SAMs on metals. Several reviews are now available in the literature. For a comprehensive review on alkanethiol monolayers on gold surfaces, we send the reader to Ref. [14].

SAMs are most commonly grown from solution, but can also be deposited from the vapor phase. By recording the kinetic of its growth, it has been shown that the formation of a SAM obeys a two-step mechanism: At the end of the first and fast (a few minutes) step, the contact angle reaches a value close to its limit, and the thickness of the layer is about 80 to $90 \%$ of its maximum value. This first step is followed by a much slower process, which may lasts up to several hours, where both the contact angle and thickness reach their final value [15]. The first step can be describe as a diffusion controlled chemisorptions; its kinetic strongly depends on the concentration of reacting molecules in the solution, and is governed by the chemical reaction of the reactive group with the surface. The activation energy of the reaction may

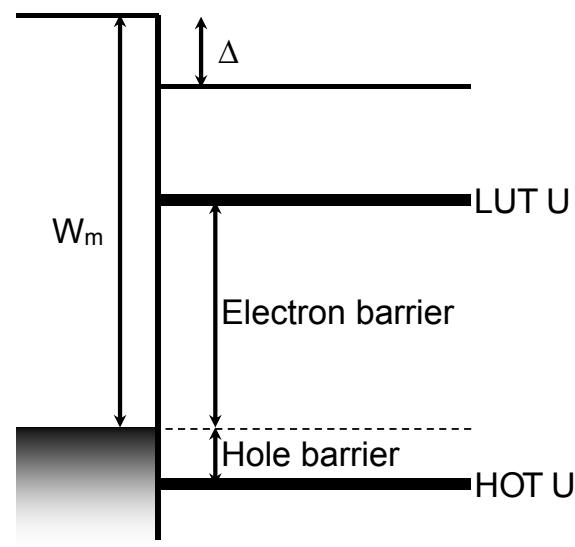

Fig. (4). Illustration of the Fermi level pinning occurring at a metal-semiconductor interface when the Fermi level of the metal approaches the LUMO (left) or HOMO (right) level. In both cases, a dipole forms at the interface, which shifts the vacuum level by an energy $\Delta$. 


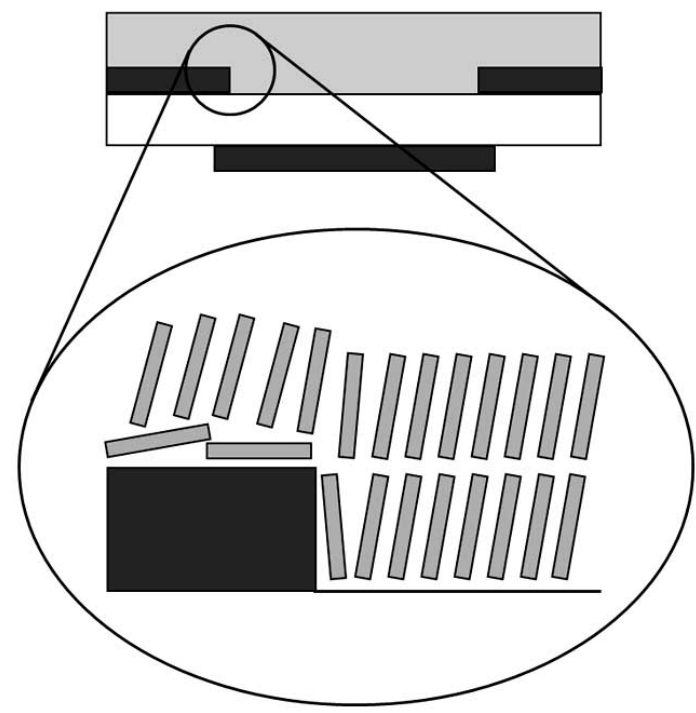

Fig. (5). Illustration of the effect of the configuration of the substrate on the morphology of the semiconductor film in a bottomcontact organic transistor.

also depend on the electron density of the adsorbing sulfur. The second step can be viewed as a crystallization process, where the alkyl chains, initially disordered, tend to form a $2 \mathrm{D}$ array. It is noteworthy that the speed of the second step increases with the length of the alkyl chain, which is interpreted in terms of increased interchain van der Walls interaction.

The chemisorption of an alkylthiol on gold may be formally considered as an oxidative addition of the $\mathrm{SH}$ group to the gold surface, followed by a reductive elimination of the hydrogen. In the case of a clean gold surface, the protons most probably end in $\mathrm{H}_{2}$ molecules, as can be inferred from the fact that monolayers can also been formed from the gas phase in the complete absence of oxygen [16]. From this simplified picture, it is clear that the formation of a SAM induces that of surface dipoles, which will play a key role in the action of the SAM in an organic transistor.

A crucial aspect of SAMs on gold and other transition metals is their stability. The thermal stability of alkanethiol SAMs has been studied by a large number of research groups. Their thermal desorption has been reported to occur at relatively high temperatures, in the range $170-230{ }^{\circ} \mathrm{C}$ [17]. As a general rule, alkanethiol SAMs are quite stable and can form a robust barrier against the oxidation or corrosion of the metal. For example, silver surfaces covered with alkanethiol monolayers could be kept in ambient atmosphere without showing any tarnishing for many months. Similarly, SAM protected copper surfaces are not attacked by nitric acid [15].

\section{Measuring the Contact Resistance}

The most widespread technique to estimate contact resistances is the so-called Transfer Line Method (TLM), which consists of measuring the transfer characteristic in the linear regime of several devices with various channel lengths [8, 18-21]. The basic concept that supports the TLM is that in a real device, the channel resistance $R_{\text {channel }}$ is in series with an additional resistance that mirror the charge injection process at the source electrode. Calling $R_{C}$ this additional resistance, and using (1) to estimate the channel resistance in the linear regime, the total resistance $R_{O N}$ is given by:

$$
\mathrm{R}_{\mathrm{ON}}=\mathrm{R}_{\mathrm{C}}+\frac{\mathrm{L}}{\mathrm{W} \mu \mathrm{C}_{\mathrm{i}}\left(\mathrm{V}_{\mathrm{G}}-\mathrm{V}_{\mathrm{T}}\right)} .
$$

What Equation (3) teaches us is that plotting the total resistance of the device as a function of the channel length should give a straight line whose extrapolation to zero length would correspond to the contact resistance, while the slope of the line can be used to estimate the mobility.

More reliable, yet more difficult to implement, is the four-probe technique that consist of introducing to additional electrodes between the source and the drain [22-24]. The principle of the technique is illustrated in Fig. (6), where $\Delta V_{S}$ and $\Delta V_{D}$ are the source and drain potential drops due to contact resistance, respectively.

A major advantage of the four-probe technique is that it allows for separately determining the source and draining contact resistances. Its main difficulty is to avoid any disturbance of the potential distribution between sources and drain when patterning the two additional electrodes [25]. This is generally done by making electrodes that hardly penetrate into the channel region, as shown in the left of Fig. (6).

\section{Morphological vs. Dipolar Effects}

Energy level alignment is the leading feature that controls charge injection in organic electroluminescent diodes and photovoltaic cells. This is because in a diode structure, the area of the contacts largely dominates over the thickness of the device, so that charge injection prevails over charge transport within the bulk of the device. In that case, the presence of an interface dipole most often prevents easy tuning

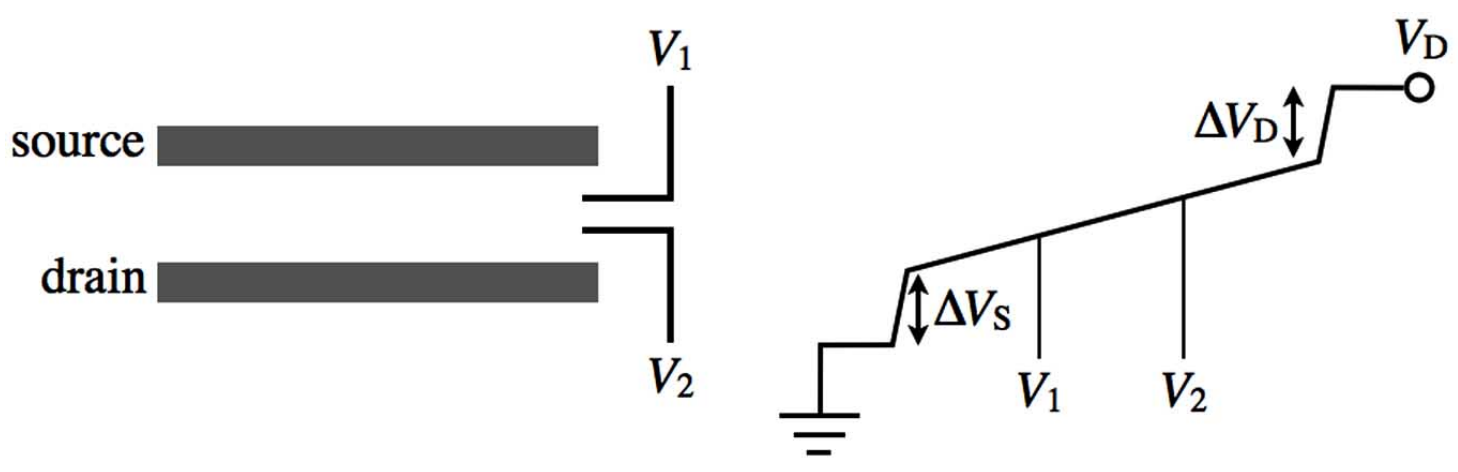

Fig. (6). Principle of the four-probe technique. Left: Image of the four electrodes; right: Potential diagram along a line perpendicular to the source and drain electrodes. 
of the injection barrier height. Early work by Campbell and coworkers has shown that an elegant way to work around this issue is to purposely introduce on top of the metal electrode a SAM bearing a counter-balancing dipole [26]. Kelvin probe was used to estimate the work function of a silver electrode modified with thiol SAMs terminated with various end groups. A decrease of the work function was observed with $\mathrm{CH}_{3}$ and $\mathrm{NH}_{2}$ end groups, while it increased with $\mathrm{CF}_{3}$. These results were in agreement with the calculated dipolar moment of the respective molecules. Later, another group confirmed these predictions by fabricating and characterizing diodes made of a modified Ag electrode and MEH-PPV, a derivative of polyphenylenevinylene, as the semiconductor [27]. With the $\mathrm{CH}_{3}$ terminated SAM, the work function of silver was lowered down to $3.8 \mathrm{eV}$, and the hole current of the diode was completely blocked, while with the $\mathrm{CF}_{3}$ end group, the work function of the electrode rose up to $5.5 \mathrm{eV}$ and the current of the diode increased by six orders of magnitudes at $5 \mathrm{~V}$ bias.

No improvement of that amplitude has been reported in organic transistors, which has been attributed to the very different geometry as compared to that of the diode. Instead, the effect of SAM is often attributed to morphological effects. Recent works by Bock and coworkers seem to corroborate early results going in that direction $[11,12]$. In this case, the SAMs were made of thiols with an anthracene end group. Interestingly, TLM measurements did not show any improvement of charge injection; instead, the channel resistance decreased by a factor of ten. On the basis of temperature dependent data, the authors went to the conclusion that the improved charge transport had to be attributed to a strong reduction of the density of traps.

However, other results seem to indicate that barrier lowering through the mechanism described by Campbell does also lead to a reduction of contact resistance in transistors, yet at a lesser degree than that observed in diodes. First, a Dutch group reported on transistors made of MEH-PPV and regio-regular polyhexylthiophene (rr-P3HT) with gold electrodes modified by decanethiol SAMs with $\mathrm{CH}_{3}$ and $\mathrm{CF}_{3}$ end groups [28]. This time, TLM measurements indicated that the channel mobility only depends on the nature of the semiconductor, not on that of the contact. As for the contact resistance, it was found to be one order of magnitude larger for the SAM-modified devices with respect to the untreated electrodes. However, the contact resistance was lower with the $\mathrm{CF}_{3}$ terminated SAMs than with the $\mathrm{CH}_{3}$ terminated SAMs.

More recently, a group in Paris reported on a similar work with pentacene as the semiconductor [29]. In this case, the TLM extracted contact resistance showed a clear increase of the contact resistance with $\mathrm{CH}_{3}$ terminated SAMs and an improved injection with the fluorinated SAMs, which is exactly what expected from the respective work functions of the modified gold electrode. Interestingly, the morphology of the pentacene on the electrode did not follow the same trend. Instead, the film showed a fibrillar structure on bare gold, indicative of molecules lying down on the substrate [9], while the structure characteristic of standing molecules was observed on both SAM-modified electrodes. In other words, there was no correlation between morphology and contact resistance. All the effect could be attributed to dipole-induced barrier tuning.

These observations have been extended to n-channel transistors, with perfectly symmetric results. That is, SAMs bearing a dipole moment pointing towards the semiconductor produce a decrease of the contact resistance, while those with a dipole moment in the other direction lead to an increase of the contact resistance [30]. Again, these experimental facts are in good agreement with the energy level alignment model.

\section{Other Surface Treatments}

Several other surface treatments have been used to improve charge injection at electrodes. In a first example, a Swiss group has realized a pentacene-based transistor were the semiconductor layer was doped with $1.5 \%$ of tetrafluoro-tetracyano-quinodimethane (F4TCNQ), resulting in a reduction of the contact resistance by a factor of 20 [31]. The improvement is explained in terms of doping of the semiconductor, leading to an easier injection of holes by a mechanism of field emission, a well-documented process in conventional microelectronics.

In a second example, a thin layer of oxide was grown on top of gold source and drain electrodes by a UV-ozone treatment, also leading to a substantial reduction of the contact resistance. This counter-intuitive result was rationalized by a reduction of the injection barrier, while hole can tunnel through the thin oxide layer [32].

An even more puzzling result was reported by a group at the University of Singapore [33]. The gold electrode treatment that led to the reduction of contact resistance simply involved dipping the electrodes into Piranha solution prior to the deposition of the semiconductor layer. In this case, an analysis of the transport characteristics suggests that the improvement is due to a doping of the semiconductor close to the electrode interface.

\section{CONCLUSION}

The most widespread technique to improve charge injection at the source electrode of organic transistors with bottom contact structure is the modification of the electrode by a self-assembled monolayer prior to the deposition of the semiconductor layer. The interpretation have been put forward to account for the improvement: (1) The presence of the SAM may lead to a better morphological structure of the semiconductor; (2) The enhanced charge carrier injection comes from a better energy level alignment at both sides of the electrode-semiconductor interface. Recent investigations seem to suggest that the latter is the prevalent element. Other surface treatments have been investigated, with in some cases an impressive improvement obtained with very simple processes. In conclusion, much work remains to be done in this field.

\section{REFERENCES}

[1] Yang Y, Wudl F. Organic Electronics: From Materials to Devices. Adv Mater 2009; 21: 1401-3.

[2] Facchetti A, Mushrush M, Katz HE, Marks TJ. n-Type building blocks for organic electronics: a homologous family of fluorocar- 
bon-substituted thiophene oligomers with high carrier mobility. Adv Mater 2003; 15: 33-8.

[3] Yan $\mathrm{H}$, Chen $\mathrm{Z}$, Zheng $\mathrm{Y}$, et al. A high-mobility electrontransporting polymer for printed transistors. Nature 2009; 457: 67986.

[4] Zaumseil J, Sirringhaus H. Electron and ambipolar transport in organic field-effect transistors. Chem Rev 2007; 107: 1296-323.

[5] Ishii H, Sugiyama K, Ito E, Seki K. Energy level alignment and interfacial electronic structures at organic metal and organic organic interfaces. Adv Mater 1999; 11: 605-25.

[6] Hwang J, Wan A, Kahn A. Energetics of metal-organic interfaces: New experiments and assessment of the field. Mater Sci Eng R 2009; 64: 1-31.

[7] Braun S, Salaneck WR, Fahlman M. Energy-Level Alignment at Organic/Metal and Organic/Organic Interfaces. Adv Mater 2009; 21: $1450-72$.

[8] Necliudov PV, Shur MS, Gundlach DJ, Jackson TN. Contact resistance extraction in pentacene thin film transistors. Sol State Electron $2003 ; 47: 259-62$.

[9] Hu WS, Tao YT, Hsu YJ, Wei DH, Wu YS. Molecular orientation of evaporated pentacene films on gold: Alignment effect of selfassembled monolayer. Langmuir 2005; 21: 2260-6.

[10] Luo Y, Gustavo F, Henry JY, et al. Probing local electronic transport at the organic single-crystal/dielectric interface. Adv Mater 2007; 19: 2267-73.

[11] Bock C, Pham DV, Kunze U, Kafer D, Witte G, Woll C. Improved morphology and charge carrier injection in pentacene field-effect transistors with thiol-treated electrodes. J Appl Phys 2006; 100: 114517.

[12] Bock C, Pham DV, Kunze U, Kafer D, Witte G, Terfort A. Influence of anthracene-2-thiol treatment on the device parameters of pentacene bottom-contact transistors. Appl Phys Lett 2007; 91: 052110 .

[13] Kymissis I, Dimitrakopoulos CD, Purushothaman S. Highperformance bottom electrode organic thin-film transistors. IEEE Trans Electron Device 2001; 48: 1060-4.

[14] Ulman A. An introduction to ultrathin organic films: From Langmuir-Blodgett to self-assembly. Academic Press: San Diego; 1991.

[15] Ulman A. Formation and structure of self-assembled monolayers. Chem Rev 1996; 96: 1533-54.

[16] Chailapakul O, Sun L, Xu C, Crooks RM. Interactions between organized, surface-confined monolayers and vapor-phase probe molecules. 7. Comparison of self-assembling n-alkanethiol monolayers deposited on gold from liquid and vapor phases. J Am Chem Soc 1993; 115: 12459-67.

[17] Schlenoff JB, Li M, Ly H. Stability and Self-Exchange in Alkanethiol Monolayers. J Am Chem Soc 1995; 117: 12528-36.

[18] Blanchet GB, Fincher CR, Lefenfeld M, Rogers JA. Contact resistance in organic thin film transistors. Appl Phys Lett 2004; 84: 296-8.
[19] Gundlach DJ, Zhou L, Nichols JA, Jackson TN, Necliudov PV, Shur MS. An experimental study of contact effects in organic thin film transistors. J Appl Phys 2006; 100: 024509.

[20] Klauk H, Schmid G, Radlik W, et al. Contact resistance in organic thin film transistors. Sol State Electron 2003; 47: 297-301.

[21] Zaumseil J, Baldwin KW, Rogers JA. Contact resistance in organic transistors that use source and drain electrodes formed by soft contact lamination. J Appl Phys 2003; 93: 6117-24.

[22] Chwang AB, Frisbie CD. Field effect transport measurements on single grains of sexithiophene: Role of the contacts. J Phys Chem B 2000; 104: 12202-9.

[23] Gelinck GH, Geuns TCT, de Leeuw DM. High-performance allpolymer integrated circuits. Appl Phys Lett 2000; 77: 1487-9.

[24] Yagi I, Tsukagoshi K, Aoyagi Y. Direct observation of contact and channel resistance in pentacene four-terminal thin-film transistor patterned by laser ablation method. Appl Phys Lett 2004; 84: 8135.

[25] Pesavento PV, Puntambekar KP, Frisbie CD, McKeen JC, Ruden PP. Film and contact resistance in pentacene thin-film transistors: Dependence on film thickness, electrode geometry, and correlation with hole mobility. J Appl Phys 2006; 99: 094504.

[26] Campbell IH, Rubin S, Zawodzinski TA, et al. Controlling Schottky energy barriers in organic electronic devices using selfassembled monolayers. Phys Rev B 1996; 54: 14321-4.

[27] de Boer B, Hadipour A, Mandoc MM, Van Woudenbergh T, Blom PWM. Tuning of metal work functions with self-assembled monolayers. Adv Mater 2005; 17: 621-5.

[28] Asadi K, Gholamrezaie F, Smits ECP, Blom PWM, de Boer B. Manipulation of charge carrier injection into organic field-effect transistors by self-assembled monolayers of alkanethiols. J Mater Chem 2007; 17: 1947-53.

[29] Marmont P, Battaglini N, Lang P, et al. Improving charge injection in organic thin-film transistors with thiol-based self-assembled monolayers. Org Electron 2008; 9: 419-24.

[30] Boudinet D, Blevennec GL, Serbutoviez C, Verilhac J-M, Yan H, Horowitz G. Contact resistance and threshold voltage extraction in n-channel organic thin film transistors on plastic substrates. J Appl Phys 2009; 105: 084510.

[31] Vanoni C, Tsujino S, Jung TA. Reduction of the contact resistance by doping in pentacene few monolayers thin film transistors and self-assembled nanocrystals. Appl Phys Lett 2007; 90: 193119.

[32] Stadlober B, Haas U, Gold H, et al. Orders-of-magnitude reduction of the contact resistance in short-channel hot embossed organic thin film transistors by oxidative treatment of Au-electrodes. Adv Funct Mater 2007; 17: 2687-92.

[33] Cai QJ, Chan-Park MB, Zhang J, et al. Bottom-contact poly(3,3"'didodecylquaterthiophene) thin-film transistors with reduced contact resistance. Org Electron 2008; 9: 14-20.

(C) Gilles Horowitz; Licensee Bentham Open .

This is an open access article licensed under the terms of the Creative Commons Attribution Non-Commercial License (http://creativecommons.org/licenses/by-nc/3.0/) which permits unrestricted, non-commercial use, distribution and reproduction in any medium, provided the work is properly cited. 